\title{
Viability and Infectivity of Cryptosporidium parvum Oocysts Detected in River Water in Hokkaido, Japan
}

\author{
Yoshinori TSUSHIMA ${ }^{1,2)}$, Panagiotis KARANIS ${ }^{3)}$, Takenori KAMADA ${ }^{1)}$, Xuenan XUAN ${ }^{1)}$, \\ Levi H. C. MAKALA ${ }^{1)}$, Yukinobu TOHYA ${ }^{2)}$, Hiroomi AKASHI ${ }^{2)}$ and Hideyuki NAGASAWA ${ }^{1) *}$ \\ ${ }^{1)}$ National Research Center for Protozoan Diseases, Obihiro University, Inada-cho, Obihiro, Hokkaido 080-8555, ${ }^{2)}$ Department of \\ Veterinary Microbiology, Graduate School of Agricultural and Life Science, The University of Tokyo, 1-1-1 Yayoi, Bunkyo-ku, Tokyo \\ 113-8657, Japan and ${ }^{3)}$ Clinical-Research Laboratory Medical School, University of Cologne Center of Anatomy, Institute II Joseph- \\ Stelzmann-Str. 9, D-50931 Cologne, Germany
}

(Received 1 August 2002/Accepted 18 January 2003)

\begin{abstract}
The viability and infectivity of Cryptosporidium parvum (C. parvum) oocysts, detected in water samples collected from river water in Hokkaido, were investigated using Severe Combined Immunodeficient (SCID) mice. The water samples collected from September 27 through October 10, 2001 by filtration using Cuno cartridge filters were purified and concentrated by the discontinuous centrifugal flotation method. From $1.2 \times 10^{5}$ liters of the raw river water, approximately $2 \times 10^{4}$ oocysts were obtained and designated as Hokkaido river water 1 isolate (HRW-1). Oocyst identification was carried out using microscopic and immunological methods. Six 8week-old female SCID mice were each inoculated orally with $1 \times 10^{3}$ oocysts. Infection was successfully induced, resulting in fecal oocyst shedding. Oocysts were then maintained by sub-inoculation into SCID mice every 3 months. Infectivity was evaluated by making comparisons with two known C. parvum stocks, HNJ-1 and TK-1, which were bovine genotypes detected in fecal samples from a cryptosporidiosis patient and young cattle raised in Tokachi, Hokkaido respectively. The oocyst genotypes were determined from a small subunit ribosomal RNA (SSU-rRNA) gene by polymerase chain reaction - restriction fragment length polymorphism (PCR-RFLP) analysis. No significant differences were observed in the average number of oocysts per gram of feces (OPG) in any of the isolates. Our data indicates that the C. parvum oocysts detected in the sampled river water were of C. parvum genotype 2 . Moreover, our data on the continued isolation, detection and identification of the $C$. parvum isolates is consistent with the available epidemiological data for the Tokachi area.
\end{abstract}

KEY WORDS: Cryptosporidium parvum oocyst, infectivity, SCID mouse, viability, water.

Cryptosporidiosis is recognized as one of the main causes of diarrhea in immunologically healthy and immunocompromised humans and animals of veterinary importance throughout the world. However, it was only after the 1983 outbreak in Texas [3] that cryptosporidiosis became known as a common disease that infects humans drinking contaminated tap water. The symptoms of Cryptosporidium infection are diarrhea, stomachache, nausea, fever and fatigue. It has been reported that the average pre-patent period ranges from 1 to 12 days in humans [4]. Because of the variable pre-patent period, identification of the cause of outbreaks is usually difficult.

Since the 1980s, outbreaks have been reported in several countries and cryptosporidiosis has now been identified as one of the emerging diseases in the last two decades. During the Milwaukee cryptosporidiosis outbreak, 400,000 cases were reported [9]. Two outbreaks of waterborne cryptosporidiosis have occurred in Japan. The first was reported at Hiratsuka in Kanagawa prefecture in 1994 with about 500 cases and the second at Ogose in Saitama prefecture in 1996 with 8,000 cases. Previously, environmental waters in Japan were considered cleaner and safer than in other countries. However, the waterworks system in Japan appears to

\footnotetext{
* Correspondence to: Nagasawa, H., National Research Center for Protozoan Diseases, Obihiro University, Inada-cho, Obihiro, Hokkaido 080-8555, Japan.
}

have entered a critical phase. Recently, in 2002, two outbreaks occurred in Hokkaido. The need to promptly carry out measures to deal with the impending problem of cryptosporidiosis in Hokkaido cannot be over emphasized.

Monitoring of Cryptosporidium oocysts is one of the most important steps in dealing with cryptosporidiosis. In monitoring Cryptosporidium oocysts, it is important that species are determined. In view of the potential harm to humans, it is especially important to investigate the genotype, viability and infectivity of Cryptosporidium species in environmental waters.

Studies have involved various polymorphic gene loci of the two major genotypes of $C$. parvum in humans $[1,2,11$, $14,16,23]$. Genotype 1, or the human genotype of $C$. par$v u m$, has been isolated almost exclusively from humans and is associated mainly with anthroponotic (human to human) transmission cycles [15]. Experiments using animals such as cattle and mice infected with the human genotype have been unsuccessful, and the only in vivo model that exists for this genotype is a gnotobiotic piglet model [22]. So far, the only reported animals infected with $C$. parvum genotype 1 are monkeys in the United States [19] and dugongs (Dugong dugon) in Australia [12]. In contrast, genotype 2 or the bovine genotype of $C$. parvum infects a wide range of mammals, including humans $[14,16]$. Genotype 2 isolates have been associated with the zoonotic (animal to human) trans- 
mission cycle. Furthermore, the genotype 2 isolates were infective to Severe Combined Immunodeficient (SCID) mice or calves. However, genotype 1 isolates were not [15].

Recently, various species of Cryptosporidium and genotypes of $C$. parvum were determined using large-scale genomic sequencing. Taking into account the pathogenicity and infectivity to humans, this study aimed at examining whether the oocysts detected in river water were $C$. parvum and whether the detected $C$. parvum oocysts were genotype 1 or 2, using microscopic and DNA based techniques [24].

\section{MATERIALS AND METHODS}

Oocysts: Three stocks of C. parvum oocysts were used in this study. The first stock, designated Hokkaido River Water - 1 (HRW-1), was detected and isolated in the Obihiro River in Hokkaido using microscopic and immunological techniques. From September 27 through October 10, 2001 , approximately $1.2 \times 10^{5}$ liters of river water was filtered and $2 \times 10^{4}$ oocysts were detected by indirect an immuno-fluorescence antibody test (IFAT) using the IFAT kit (Cellabs DIF, Cellabs Pty. Ltd., Sydney/Australia) and 4', 6-diamidino-2-phenylindole (DAPI).

The second stock, TK-1, was obtained from fecal samples of cattle raised in the Tokachi area [18]. The third stock, HNJ-1, was $C$. parvum genotype 2 detected in fecal samples from a patient with cryptosporidiosis [10]. All stocks and strains were maintained by sub-inoculation into SCID mice every three months.

Collection, purification and concentration of oocysts from river water: Collection, purification and concentration of oocysts from river water were carried out using previously described methods [20]. Briefly, filtration using a Cuno cartridge filter, the discontinuous sucrose gradient method, and IFAT and DAPI staining were carried out. After staining, samples were analyzed using a UV-light microscope. The UV excitation bands ranged from 450 to $490 \mathrm{~nm}$ for IFAT staining and from 310 to $395 \mathrm{~nm}$ for DAPI staining. The number of oocysts was counted and then the oocysts were stored in $2.5 \%$ (w/v) solution of $\mathrm{K}_{2} \mathrm{Cr}_{2} \mathrm{O}_{7}$ until further use.
Mouse infectivity: Six 8-week-old female SCID mice (the $\mathrm{CP}$ group) were each orally inoculated with $\times 10^{3} \mathrm{C}$. parvum oocysts from the river water. The mice were each raised in their own wire netted cage. Feces were collected every two days starting on Day 2 post infection. The oocysts were purified using the sucrose floatation method as previously reported [8] and identified using IFAT and DAPI staining. Oocysts per gram of feces (OPG) were recorded for 30 days. On Day 30 post infection, the mice were sacrificed, and their intestines removed and the contents examined by IFAT staining.

Three parallel experiments were conducted involving three groups of 6 8-week-old female SCID mice, the HRW$1, \mathrm{HNJ}-1$ and TK-1 groups. Depending on the group, each mouse was orally inoculated with $1 \times 10^{4}$ oocysts from either the HRW-1, HNJ-1 or TK-1 stock. The mice were treated and the oocysts investigated as in the above experiment.

PCR-RFLP analysis: Oocyst genotype was determined using the PCR-RFLP analysis for a small subunit ribosomal RNA (SSU-rRNA) gene as previously reported [33]. Oocyst species determination was carried out by digesting the secondary PCR product with the Ssp I. C. parvum generated three visible bands at 448, 247 and $106 \mathrm{bp}$. To differentiate human and bovine genotypes of $C$. parvum, the secondary PCR product was digested with Ase I (Vsp I). C. parvum bovine genotype exhibited two visible bands at 628 and $104 \mathrm{bp}$.

\section{RESULTS}

UV light microscopy: C. parvum oocysts detected in the river water and in feces from cattle were stained by IFAT and DAPI staining and observed under a UV light microscope. IFAT and DAPI staining of the oocysts are summarized in Fig. 1. The oocysts were spherical and $3-5 \mu \mathrm{m}$ in diameter. Moreover, they had a suture line with apple-green fluorescence when stained with IFAT as shown in Fig. 1A, and they had four sporozoite nuclei with pale fluorescence when stained with DAPI as shown in Fig. 1B. Taken together, these characteristics indicate that the isolated
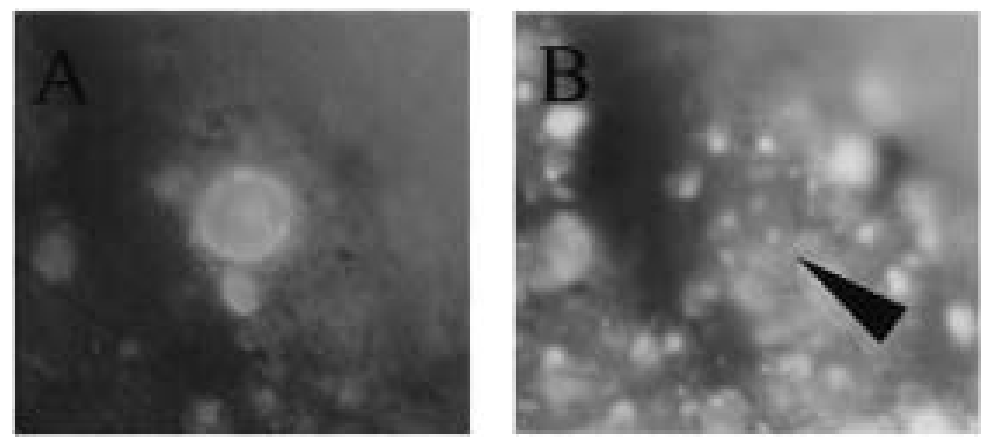

Fig. 1. C. parvum oocysts isolated from river water samples as shown by: (A) IFAT and (B) DAPI staining. Note - The apple-green color staining of the C. parvum oocysts in (A) and the 4 pale colored sporozoite nuclei (B). 


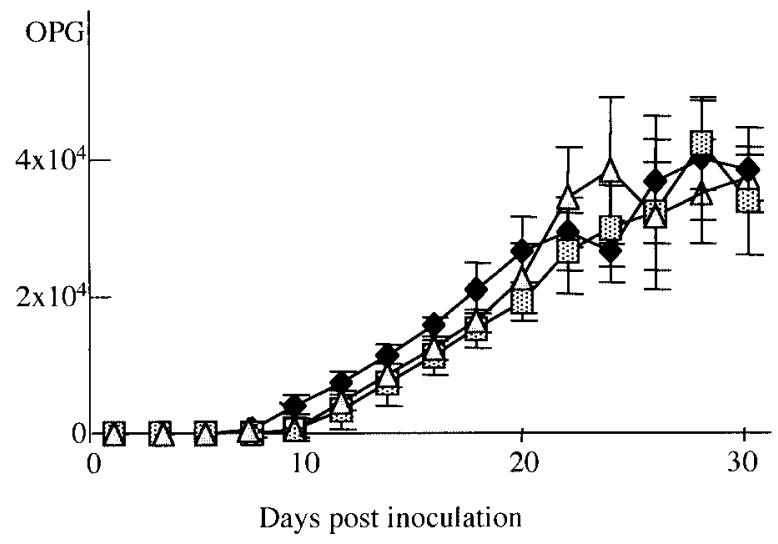

Fig. 2. OPG values from 3 groups of oocyst stocks (HNJ-1, TK-1 and HRW-1) at 2 days intervals in SCID mice orally inoculated with $1 \times 10^{4} C$. parvum oocysts. $\bullet$ : HNJ-1, $\triangle$ : HRW-1, ti: TK-1

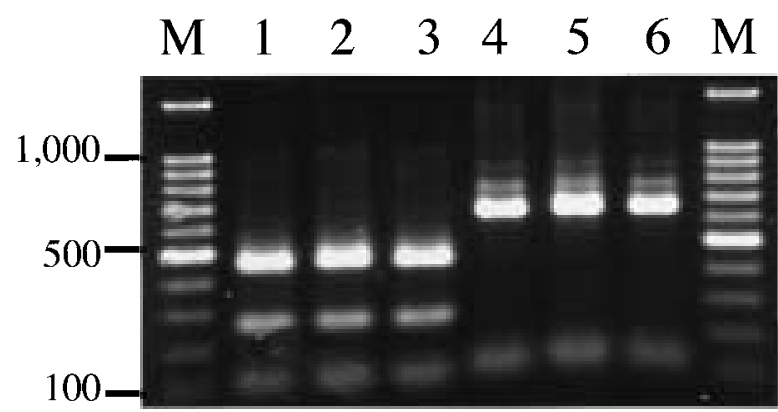

Fig. 3. Molecular diagnosis of C. parvum oocysts by PCR-RLFP procedure based on the SSU rRNA gene sequence. Species diagnoses are shown in Lane 1 to 3. C. parvum genotype differentiation is shown in Lanes 4 to 6.C. parvum bovine genotype HNJ-1 strain is shown in Lanes 1 and 4. C. parvum TK-1 stock is shown in Lanes 2 and 5. C. parvum HRW-1 stock is shown in Lanes 3 and 6.

oocysts in the river water were C. parvum.

Mouse infectivity: In the $\mathrm{CP}$ group, mouse infectivity was successful, with oocyst appearing in the feces in 2 SCID mice. The pre-patent periods were 10 and 12 days post infection. Throughout the 30 days in which the OPG was observed, the SCID mice continued to shed oocyst in their feces.

The average OPG of the feces of the HRW-1, HNJ-1 and TK-1 groups of mice is summarized in Fig. 2. No significant differences were observed in the average OPG of any of the isolates. Upon inoculation, infection was successfully induced, indicating that the isolated oocysts were alive and infective.

PCR-RFLP analysis: Results of the PCR-RFLP analysis are shown in Fig. 3. The three samples tested, HNJ-1, TK-1 and HRW-1, generated three visible bands of 448, 247 and $106 \mathrm{bp}$, respectively, following digestion of the secondary PCR product with Ssp I (Lane 1 to 3 ). When the secondary
PCR product was digested with Ase I, two visible bands at 628 and 104 bp were generated in Lanes 4 to 6 , suggesting that oocysts detected in the water sample were of $C$. parvum genotype 2 .

\section{DISCUSSION}

Our study has shown that the $C$. parvum oocysts detected in the sampled river water in Hokkaido are viable and infective to susceptible hosts. Moreover, by using a DNA based technique, we were also able to demonstrate that the isolated oocysts belong to the bovine genotype. Our data on the continued isolation, detection and identification of the C. parvum isolates is consistent with available epidemiological data and has shown that the animal-inoculation assay used in this study is an effective tool for monitoring Cryptosporidium in environmental water.

Monitoring C. parvum oocysts in environmental water involves determining the viability, infectivity and genotype of oocyst species, in connection with their reported pathogenicity to humans.

When detected oocysts are viable, oocyst infectivity to hosts becomes an important concern. Recently, DAPI/PI staining has been used in viability assays. However, there are problems associated with DAPI/PI staining. For instance, the vast amount of debris in the water samples makes it difficult to discriminate specific fluorescence from non-specific fluorescence from the four nuclei under the UV light microscope. Identification of the 4 sporozoites in the stained nuclei was difficult, as shown in Fig. 1B.

To date, several different assays to investigate oocyst viability and infectivity have been reported in previous related studies [5]. In this study, the mouse-inoculation assay was used for two reasons. First, because it was easy to determine viability and easy to demonstrate infectivity following oocyst shedding and, second, because it increased the number of oocysts.

Infection of the isolated oocysts in SCID mice was successfully demonstrated by oocyst shedding in feces of inoculated SCID mice. The oocysts were shown to be viable and infective to the SCID mice. To compare the patterns of oocyst shedding, C. parvum HNJ-1, TK-1 and HRW-1 oocyst stocks were used. Patterns of oocyst shedding showed a similar trend in all three oocyst stocks.

The pattern of increase in oocyst numbers was similar in all three oocyst stocks. In this study, we obtained a yield of approximately $2 \times 10^{4}$ oocysts, indicating that only a small number of oocysts can be isolated from environmental waters. The small number of oocysts may make it difficult to conduct DNA and RNA based techniques that require large numbers of oocysts.

The results of PCR-RFLP analysis indicated that oocysts in river water belong to the C. parvum genotype 2. This finding is consistent with the existing and previously reported epidemiological data for the Tokachi area in Hokkaido [18]. From our results, cattle may be the source of the C. parvum polluting river water. Many types of Cryptospo- 
ridium species have been detected in feces of humans and animals [3]. More studies regarding the epidemiology of Cryptosporidium species need to be carried out in the Tokachi area of Hokkaido and in Japan as a whole.

In conclusion, the results of this study have shown that $C$. parvum oocysts detected in sampled river water in the Tokachi area of Hokkaido are viable and, therefore, may be infective to susceptible hosts, as demonstrated by sub-inoculation into SCID mice. Moreover, by using a DNA based technique, we have shown that the isolated oocysts belonged to $C$. parvum genotype 2 . Our results appear to be consistent with the current prevalence of bovine cryptosporidiosis. The success in the use of SCID mice to demonstrate $C$. parvum genotype 2 infection indicates that laboratory animals are helpful in the analysis of viability and infectivity. Recently, it has been reported that $C$. parvum genotype 1 does not infect SCID mice [16] and that $C$. parvum genotype 1 can infect not only humans but also pigs [15]. In the laboratory, gnotobiotic pigs were used for maintaining C. parvum genotype 1 oocyst [1]. Studies have shown that $C$. parvum genotype 1 infects not only humans but also pigs, primates, and other animals, making the identification of the cause of $C$. parvum genotype 1 outbreaks difficult [16]. Further studies need to be carried out to investigate the application of other laboratory animals, for example, pigs, in order to develop monitoring systems for C. parvum genotype 1 .

Our results also send a warning that the Tokachi area of Hokkaido, Japan may harbor a significant presence of $C$. parvum oocysts capable of transmitting infection to human hosts. In view of the two recent outbreaks reported in Hokkaido in 2002, it is imperative that prompt measures be taken to deal with the impending threat of human cryptosporidiosis.

AKNOWLEDGEMENTS. The authors are profoundly grateful to Professor Dr. Iseki and Professor Dr. Sarashina for kindly providing C. parvum isolates and helpful advice. This study was supported by Grants-in-Aid for Scientific Research from the Ministry of Education, Culture, Sports, Science and Technology of Japan.

\section{REFERENCES}

1. Awad-El-Kariem, F. M. 1999. Does Cryptosporidium parvum have a clonal population structure? Parasitol. Today 15: 502504.

2. Caccio, S., Homan, W., Camilli, R., Traldi, G., Kortbeek, T. and Pozio, E. 2000. A microsatellite marker reveals population heterogeneity within human and animal genotypes of Cryptosporidium parvum. Parasitology 120: 237-244.

3. D'Antonio, R. G. 1995. A waterborne outbreak of cryptosporidiosis in normal hosts. Annals. Int. Med. 103: 886-888.

4. DuPont, H. L., Chappell, C. L., Sterling, C. R., Okhuysen, P. C., Rose, J. B. and Jakubowski, W. 1995. The infectivity of Cryptosporidium parvum in healthy volunteers. New Engl. J. Med. 332: 855-859.

5. Fayer, R., Morgan, U. and Upton, S. J. 2000. Epidemiology of Cryptosporidium: transmission, detection and identification.
Int. J. Parasitol. 30: 1305-1322.

6. Jenkins, M. B., Anguish, L. J., Bowman, D. D., Walker, M. J. and Ghiorse, W. C. 1997. Assessment of a dye permeability assay for determination of inactivation rates of Cryptosporidium parvum oocysts. Appl. Environ. Microbiol. 63: 3844 3850 .

7. Jenkins, M. C., Trout, J., Abrahamsen, M. S., Lancto, C. A., Higgins, J. and Fayer, R. 2000. Estimating viability of Cryptosporidium parvum oocysts using reverse transcriptasepolymerase chain reaction (RT-PCR) directed at mRNA encoding amyloglucosidase. J. Microbiol. Methods 43: $97-$ 106.

8. Kimura, A., Karanis, P., Maltezos, H. and Seitz, H. M. 2000. Bench scale experiments to evaluate the usefulness of sucrose flotation techniques for separation of Cryptosporidium oocysts from water. J. Protozool. Res. 10: 155-165.

9. MacKenzie, W. R., Schell, W. L., Blair, K. A., Addiss, D. G., Peterson, D. E., Hoxie, N. J., Kazmierczak, J. J. and Davis, J. P. 1995. Massive outbreak of waterborne Cryptosporidium infection in Milwaukee, Wisconsin: recurrence of illness and risk of secondary transmission. Clin. Infect. Dis. 21: 57-62.

10. Masuda, G., Negishi, M., Ajisawa, A., Yamaguchi, T., Tajima, T., Tamagawa, S., Maeda, Y., Ohtomo, H., Kimata, I., Uni, S., et al. 1991. Cryptosporidium diarrhea developing in two Japanese adults-one in AIDS and the other in a normal host. Research Group for Infectious Enteric Diseases, Japan. Kansenshogaku Zasshi. 65: 1614-1619 (in Japanese).

11. McLauchlin, J., Amar, C., Pedraza-Diaz, S. and Nichols, G. L. 2000. Molecular epidemiological analysis of Cryptosporidium spp. in the United Kingdom: results of genotyping Cryptosporidium spp. in 1,705 fecal samples from humans and 105 fecal samples from livestock animals. J. Clin. Microbiol. 38: 3984 3990.

12. Morgan, U. M., Xiao, L., Hill, B. D., O’Donoghue, P., Limor, J., Lal, A. and Thompson, R. C. 2000. Detection of the Cryptosporidium parvum "human" genotype in a dugong (Dugong dugon). J. Parasitol. 86: 1352-1354.

13. Morgan, U. M., Constantine, C. C., Forbes, D. A. and Thompson, R. C. 1997. Differentiation between human and animal isolates of Cryptosporidium parvum using rDNA sequencing and direct PCR analysis. J. Parasitol. 83: 825-830.

14. Ong, C. S., Eisler, D. L., Goh, S. H., Tomblin, J., Awad-ElKariem, F. M., Beard, C. B., Xiao, L., Sulaiman, I., Lal, A., Fyfe, M., King, A., Bowie, W. R. and Isaac-Renton, J. L. 1999. Molecular epidemiology of cryptosporidiosis outbreaks and transmission in British Columbia, Canada. Am. J. Trop. Med. Hyg. 61: 63-69.

15. Peng, M. M., Xiao, L., Freeman, A., Arrowood, M. J., Escalante, A. A., Weltman, A. C., Ong, C. S., MacKenzie, W. R., Lal, A. A. and Beard, C. B. 1997. Genetic polymorphism among Cryptosporidium parvum isolates: evidence of two distinct human transmission cycles. Emerg. Infect. Dis. 3: 567 573.

16. Pieniazek, N. J., Bornay-Llinares, F. J., Slemenda, S. B., Da Silva, A. J., Moura, I. N., Arrowood, M. J., Ditrich, O. and Addiss, D. G. 1999. New Cryptosporidium genotypes in HIVinfected persons. Emerg. Infect. Dis. 5: 444-449.

17. Robertson, L. J., Campbell, A. T. and Smith, H. V. 1998. Viability of Cryptosporidium parvum oocysts: assessment by the dye permeability assay. Appl. Environ. Microbiol. 64: 3544 3545 .

18. Sakai, H., Tsushima, T., Nagasawa, H., Tanabe, S., Uzuka, Y. and Sarashina, T. 2002. Cryptosporidium infection of cattle in 
Tokachi district, Hokkaido. J. Vet. Med. Sci. (in press).

19. Spano, F., Putignani, L., Crisanti, A., Sallicandro, P., Morgan, U. M., Le Blancq, S. M., Tchack, L., Tzipori, S. and Widmer, G. 1998. Multilocus genotypic analysis of Cryptosporidium parvum isolates from different hosts and geographical origins. J. Clin. Microbiol. 36: 3255-3259.

20. Tsushima, Y., Karanis, P., Kamada, T., Nagasawa, H., Xuan, X., Igarashi, I., Fujisaki, K., Takahashi, E. and Mikami, T. 2001. Detection of Cryptosporidium parvum oocysts in environmental water in Hokkaido, Japan. J. Vet. Med. Sci. 63: 233236.

21. Vesey, G., Ashbolt, N., Fricker, E. J., Deere, D., Williams, K. L., Veal, D. A. and Dorsch, M. 1998. The use of a ribosomal RNA targeted oligonucleotide probe for fluorescent labelling of viable Cryptosporidium parvum oocysts. J. Appl. Microbiol.
85: $429-440$

22. Widmer, G., Akiyoshi, D., Buckholt, M. A., Feng, X., Rich, S. M., Deary, K. M., Bowman, C. A., Xu, P., Wang, Y., Wang, X., Buck, G. A. and Tzipori, S. 2000. Animal propagation and genomic survey of a genotype 1 isolate of Cryptosporidium parvum. Mol. Biochem. Parasitol. 108: 187-197.

23. Xiao, L., Bern, C., Limor, J., Sulaiman, I., Roberts, J., Checkley, W., Cabrera, L., Gilman, R. H. and Lal, A. A.2001. Identification of 5 types of Cryptosporidium parasites in children in Lima, Peru. J. Infect. Dis. 183: 492-497.

24. Xiao, L., Escalante, L., Yang, C., Sulaiman, I., Escalante, A. A., Montali, R. J., Fayer, R. and Lal, A. A. 1999. Phylogenetic analysis of Cryptosporidium parasites based on the small-subunit rRNA gene locus. Appl. Environ. Microbiol. 65: 15781583. 